\title{
Fiber Optic Raman Sensor to Monitor Concentration Ratio of Nitrogen and Oxygen in a Cryogenic Mixture
}

\author{
Vidhu S.Tiwari ${ }^{1}$, Rajamohan R. Kalluru ${ }^{2}$, Fang-Yu Yueh ${ }^{1}$, and Jagdish P Singh ${ }^{1 *}$ \\ and William St. $\mathrm{Cyr}^{3}$ \\ ${ }^{1}$ Institute for Clean Energy Technology, Mississippi State University \\ 205 Research Boulevard, Starkville, MS- 39759 \\ ${ }^{2}$ Mississippi Ethanol LLC, Winona, MS- 38968 \\ ${ }^{3} N A S A /$ Stennis Space Center (SSC), SSC, MS-39529 \\ ${ }^{*}$ Corresponding Author: singh@ICET.msstate.edu
}

A spontaneous Raman scattering optical fiber sensor is developed for a specific need of NASA/SSC for long-term detection and monitoring of the quality of liquid oxygen (LOX) in the delivery line during ground testing of rocket engines. The sensor performance was tested in the laboratory and with different excitation light sources. To evaluate the sensor performance with different excitation light sources for the LOX quality application, we have used the various mixtures of liquid oxygen and liquid nitrogen as samples. The study of the sensor performance shows that this sensor offers a great deal of flexibility and provides a cost effective solution for the application. However, an improved system response time is needed for the real-time, quantitative monitoring of the quality of cryogenic fluids in harsh envioronment.

OCIS codes: $0300.6450,300.6330,300.0300,140.3550,170.5660,120.0120,60.2370$ 


\section{Introduction}

The environment within a rocket engine is particularly severe with very high temperatures and pressures along with extremely rapid fluid and gas flows. ${ }^{1}$ The escalating use of liquid phase compounds like oxygen, hydrogen, etc., as cryogenic fuels and their quality monitoring led to the development of a variety of sensor systems. Apart from quality control, sensors are required for quantitative determination of constituent species in a sample mixture, especially in aircraft fuel tanks where a disproportionate concentration of one sample against another can lead to an

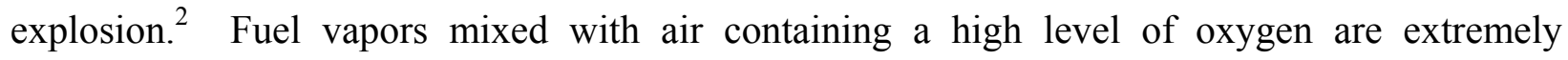
flammable and raise serious questions on the survivability of aircraft. Implementation of sensors is not only restricted to realizing ignition susceptibility but also in monitoring the health of rocket engines, especially where an optimum level of cryogenic fuel purity is highly desirable. Several types of sensors like electronic sensors, capacitance based, electrochemical sensors, etc., are commercially available but lose their relevance in the supercritical environment of rockets and aircraft, where high temperature and pressure severely inhibit their optimal performance. ${ }^{3-5}$ In the past, researchers have developed computational-analytical techniques for quality maintenance in cryogenic fuel like liquid methane at the entrance to the article tank. ${ }^{6}$ Though interesting, this method requires sampling of fuel mixture and thus fails to provide real-time measurement of impurities in fuel. Application of fiber optic technology to rocket engine health monitoring offers an alternative approach towards realizing various parameters of interest and addresses several measurement issues associated with extremely harsh environments. Today, optical fiber sensing technology has evolved to the point where one can measure nearly all the physical parameters of interest and a very large number of chemical species as well. ${ }^{7}$ 
This paper reports an all-optical cryogenic fluid sensor based on Raman spectroscopy for concentration ratio monitoring of liquid nitrogen $\left(\mathrm{LN}_{2}\right)$ in liquid oxygen (LOX). The present work is motivated by a specific need of NASA Stennis Space Center (SSC) for long-term detection and monitoring of the quality of liquid oxygen in the delivery line during the testing of rocket engines. ${ }^{8}$ An attractive design feature of a fiber optic sensor lies in real-time, in-situ qualitative as well as quantitative determination of $\mathrm{LOX}$ and/or $\mathrm{LN}_{2}$ from their mixture sample on the basis of spectrum analysis for various sample compositions. Prior to this study, efforts have been made to develop a gas sensor for monitoring gaseous phase mixtures of nitrogen and oxygen. ${ }^{9}$ Online characterization of cryogenic fluid mixtures like liquid nitrogen and liquid oxygen by optical diagnostics is an area that has not yet been explored to the best of our knowledge. Various issues involved in monitoring super critical fluid mixtures, like sample vaporization/condensation, system optimization, signal/noise ratio and other safety parameters, have been well addressed and provide a novel vision for encountering challenges involved in processing/controlling cryogenic fuels.

Certain distinct advantages of optical fiber sensors over conventional sensors, like remote sensing, multiplexing and distributed sensing, immunity towards EM signals, etc., make them more advantageous in real field applications. On the other hand, laser Raman spectroscopy (LRS) has been known for years as a relatively simple analytical method for identification of molecules in gases, liquids and solids by scattering of laser light. Raman spectroscopy is based on the Raman effect that results from energy exchange between incident photons and the scattered molecules. ${ }^{10-21}$ The Raman scattered light occurs at frequencies that are shifted from the incident laser light by the change in vibrational, rotational or electronic energies of a molecule. By measuring the frequency and intensity of inelastically scattered light from the 
sample, the molecules in the sample can be qualitatively and quantitatively measured. In order to explore various possibilities of employing a low cost, portable optical sensor based on LRS in the harsh environment of a rocket engine, we evaluated three excitation light sources for the sensor. Two frequency doubled 532-nm continuous wavelength (CW) Nd:YAG lasers $\left(\mathrm{TEM}_{00}\right.$ and Multimode) were first used as excitation light source with a fiber optic state-of-the-art miniaturized Raman probe. Then a diode laser operating at $670-\mathrm{nm}$ was used as the excitation source in an attempt towards configuring a cost effective optical sensor, which caters to the needs of various national laboratories and cryogenic industries. The sensor performances with different excitation sources in the measurements of liquid nitrogen in liquid oxygen are compared and the results are reported in this paper.

\section{Experimental Details}

The main focus of this research effort was to develop a real-time optical fiber sensor that employs a CW 532-nm laser as an excitation light source for monitoring the concentration of $\mathrm{LN}_{2}$ in $\mathrm{LOX} / \mathrm{LN}_{2}$ liquid mixture. In the process of developing a miniaturized and cost effective optical fiber Raman sensor, we have also evaluated a diode laser as an excitation light source. The details of the excitation sources used in this study are given in Table 1.

In the initial experiment, an optical fiber Raman sensor is set up using a frequency doubled 532-nm continuous wavelength (CW) Nd:YAG laser (Coherent DPSS 532) as the excitation light source, with maximum output power at $330 \mathrm{~mW}$. The laser output energy was attenuated through proper neutral density filters in order to protect the detector from potential damage by the high intensity light. The experimental set-up is shown in Fig. 1(a). The sensor configuration employed a modified In-Photonics fiber optic state-of-the-art miniaturized Raman 
probe. This probe consisted of just two parallel optical fibers; one (90- $\mu \mathrm{m}$ core diameter) was guiding the launched light to the liquid sample mixture enclosed within the dewar, whereas the other (200- $\mu \mathrm{m}$ core diameter) was collecting the emitted Raman signal and feeding it to a USB spectrometer (USB 2000, Ocean Optics Inc.). This spectrometer had 1200 1/mm grating along with a 2048-element charge-coupled device (CCD) attached to the exit of the spectrograph, and was interfaced with a computer via a USB port. Probe performance in terms of Raman signal response and background noise level was improved by incorporating a miniaturized lens at the tip of the probe with a working distance of about 30-mm. Experiments were carried out for the multiple concentration (weight) ratios of the liquid $\mathrm{N}_{2} / \mathrm{O}_{2}$ and mixed to ensure homogeneity within the mixture. For each sample, a 3-s sampling time was used to minimize errors due to rapid phase transforming, i.e., liquid to gaseous phase nature of the sample constituents. Owing to the continuous vaporization of the supercritical liquid mixture, the greatest challenge of the experiment was to minimize the vaporization loss, and also the accuracy with which the weight ratio of the sample constituents were determined during the preparation of mixtures. In the initial experiment, a cap with a hole was mounted on the dewar. Though it allowed a clear passage for the laser light to be focused onto the liquid $\mathrm{N}_{2} / \mathrm{O}_{2}$ mixture from the probe, it could not prevent the vapors from escaping and condensing onto the tip of the fiber probe. As a result, attenuation in the laser power was observed that severely hampered the liquid $\mathrm{N}_{2} / \mathrm{O}_{2}$ spectrum, thereby reducing the signal-to-noise ratio of the Raman signal. To overcome this problem, two identical quartz windows (1/4") were introduced into the dewar cap hole to provide optical access as well as to prevent vapors from reaching the tip of the probe. A dryer was also installed in the vicinity of the fiber probe to eliminate the remote possibility of vapor deposition onto the tip of the probe and thus to achieve long-term stable operation of the Raman sensor. 
To study the effects of laser property to Raman signal, the Raman data from a narrow band 532-nm laser (Coherent DPSS 532) and a high-power and inexpensive broadband 532-nm laser (Milles Griot GHS 309) were compared. The experimental configuration was kept the same as we recorded these data. A neutral density filter was applied to the broadband 532-nm laser output to attenuate the laser power so that the laser energy on the sample mixture was about $\sim 140 \mathrm{~mW}$ from both lasers.

Later we designed a prototype Raman sensor based on a $700 \mathrm{~mW}$ power, CW diode laser (Power Technology Inc.), operating at 670-nm, as an excitation light source and a Y-shaped reflection/backscattering probe (R200-REF, Ocean Optics Inc.). Figure 1(b) shows the schematic diagram of this system. This Y-shaped probe consists of seven optical fibers, each having $200 \mu \mathrm{m}$ core diameters and 0.22 as numerical aperture with one launching fiber and six surrounding collecting fibers. Uniqueness of the prototype sensor lies in its compact design configuration that included carefully aligned optical components, viz., laser diode, filter holder, cut-off filter (725-nm) and a compact OOI spectrometer (USB 2000). Raman Spectrums of different concentration ratio mixtures of $\mathrm{LOX}$ and $\mathrm{LN}_{2}$ were recorded with the prototype Raman sensor in the same manner as described earlier.

\section{Results and Discussions}

The present study was primarily focused on quantitative as well as qualitative analysis of component ratio of liquid $\mathrm{N}_{2} / \mathrm{O}_{2}$ mixtures at varied levels of their weight ratio. The mixture sample was prepared by adding liquid $\mathrm{O}_{2}$ in liquid $\mathrm{N}_{2}$, owing to the fact that the molecular weight of oxygen is higher than nitrogen, and therefore suppressing the rate of vaporization of liquid nitrogen. ${ }^{22}$ Raman Spectrum recorded for $40 \%$ of $\mathrm{LOX}$ and $60 \%$ of $\mathrm{LN}_{2}$ mixture is shown in the Fig.2 (a). Raman bands of LOX ( 580-nm) and $\mathrm{LN}_{2}(\sim 607-\mathrm{nm})$, corresponding Raman shift for 
$\mathrm{O}_{2}$ at $1556.4 \mathrm{~cm}^{-1}$ and $\mathrm{N}_{2}$ at $2330.7 \mathrm{~cm}^{-1}$, were marked on the spectrum. ${ }^{23}$ The concentration of $\mathrm{LN}_{2}$ was varied from $1 \%$ to $100 \%$ against $\mathrm{LOX}$ at normal atmospheric pressure and temperature, while maintaining a total mixture weight of 100 gram. The Raman band intensity corresponding to $\mathrm{LN}_{2}$ was increasing as the concentration of $\mathrm{LN}_{2}$ was increased against the $\mathrm{LOX}$ in the sample mixture as shown in Fig. 2(b). A narrow band Coherent CW laser, operating at 532-nm, was used in this experiment.

The observed Raman bands of $\mathrm{LOX}$ and $\mathrm{LN}_{2}$ were critically analyzed in terms of spectral band shape, $\mathrm{S} / \mathrm{N}$ ratio, peak intensity, etc. Peak intensity corresponding to $\mathrm{LN}_{2} / \mathrm{LOX}$, at various concentrations of their weight ratio, was estimated for obtaining a calibration curve as shown in Fig. 3. Sensor response exhibits a linear trend within the concentration limit of $1 \%$ to $60 \%$ of $\mathrm{LN}_{2}$ and observed to be saturating in the concentration range of $60 \%$ to $80 \% \mathrm{LN}_{2}$ in a sample mixture. Slower response of the sensor towards a higher concentration ratio of $\mathrm{LN}_{2}$ attributes to the fact that the vaporization rate of liquid nitrogen increases manifolds resulting in the formation of a cloud of semi-liquid gaseous phase mixture; and thereby affecting the sensor performance. We further calculated the spectral band area ratios of Raman $\mathrm{LN}_{2}$ and $\mathrm{LOX}$ peaks. This calculation was performed for all the sets of spectra corresponding to a particular concentration ratio of $\mathrm{LOX} / \mathrm{LN}_{2}$, and averaged to obtain a single data point. As a result, error due to the fluctuation in the peak intensity was minimized. Finally, a calibration curve was drawn between the averaged spectral band area ratios of Raman $\mathrm{LN}_{2}$ and LOX peaks against the corresponding weight ratios as shown in Fig. 4. The following conclusions have been drawn from the experimental results obtained above.

First, the sensor system is capable of successfully monitoring the $\mathrm{LN}_{2} / \mathrm{LOX}$ level in a $\mathrm{LN}_{2} / \mathrm{LOX}$ liquid mixture. Second, the Raman peak area follows the same characteristics as the 
intensity ratio curve. For example, both intensity and area ratio curves exhibit a linear trend within a low concentration range of $\mathrm{LN}_{2}$ which tend to saturate above $60 \% \mathrm{LN}_{2}$ concentration. This implies that the sensor has high detection sensitivity in low concentration range and can measure $\mathrm{LN}_{2}$ percentages as low as $1 \%$ in a sample mixture. For a typical sample mixture of $60 \% \mathrm{LN}_{2}$ and $40 \% \mathrm{LOX}$, Limit of Detection (L.O.D.) of sensor was calculated as $\sim 1 \%$. Also, intensity/area ratio data points are least fluctuating as reflected by the error bars on the calibration curves which are barely visible in Fig. 3 and Fig. 4. This signifies not only the longterm stable operation of the sensor but also the reproducibility in the sensor response towards analyte concentration. Although the calibration curve obtained by the area ratio method involved statistical averaging of multiple sets of spectra, it did not provide better sensor response. The linear calibration data for $\mathrm{LN}_{2} / \mathrm{LOX}$ ratio indicates that the fiber optic Raman sensor has a great potential for qualitative as well as quantitative monitoring of sample constituent in supercritical $\mathrm{LN}_{2} / \mathrm{LOX}$ liquid mixtures.

To develop a less expensive sensor for this application, the feasibility of using an inexpensive, high power, broadband laser (Milles Griot) operating at 532-nm as an excitation light source was tested. Using the same laser energy as with Coherent laser $(\sim 140 \mathrm{~mW})$, and same experimental setup, spectra were recorded for various concentration ratios of $\mathrm{LOX}$ and $\mathrm{LN}_{2}$ and compared with the 532-nm Coherent laser used in the previous case. Comparative analysis of spectra recorded with two lasers suggest that in both the cases, spectra share common features and depending upon the requirement, either of these could be employed as excitation light sources. The laser line-width of a Milles Griot laser $(\sim 0.18 \mathrm{~nm})$ is higher than that of a Coherent laser $(\sim 0.00047 \mathrm{~nm})$ and showed a slightly higher spectral background in comparison with a 
Coherent laser. Also, a Milles Griot laser could be operated in the high range of power which is suitable for trace $\mathrm{LN}_{2} / \mathrm{LOX}$ monitoring.

To develop a prototype sensor using a 670-nm diode laser, we first estimated the performance of the sensor by considering the laser energy and detector response near 532 and $670 \mathrm{~nm}$. Since the Raman cross-section depends inversely on the fourth power of the laser wavelength $(\lambda),{ }^{21}$ the Raman signal is also affected by the excitation wavelength. The various parameters that can affect Raman signal are summarized in Table 2. Estimation based on these parameters (the effects of optical response from other optics at these two wavelengths are not included in this calculation) shows that the prototype sensor should give about 4.4 times less signal than with the system with more expensive 532-nm excitation source. This signal level should be good enough for this type of application. A typical spectrum of a $60 \% \mathrm{LN}_{2}$ and $40 \%$ LOX sample mixture, recorded with the prototype sensor that included a $670 \mathrm{~nm}$ laser as an excitation light source, is shown in Fig. 5(a), where the $\mathrm{LN}_{2}$ Raman peak corresponds to $\sim 795$ $\mathrm{nm}$ and the LOX peak corresponds to $\sim 749$-nm, respectively. The Limit of Detection (L.O.D.) of the prototype sensor for $60 \% \mathrm{LN}_{2}$ and $40 \% \mathrm{LOX}$ was calculated as $\sim 20 \%$ which is much higher in comparison to the L.O.D. of the sensor with a 532-nm laser $(\sim 1 \%)$ for the same concentration ratio mixture. The spectrum recorded with the $670-\mathrm{nm}$ laser diode also shows a strong background that severely affects the Raman signal of $\mathrm{LOX} / \mathrm{LN}_{2}$. The overall spectral quality (signal-to-noise) of the prototype sensor using a 670-nm diode laser is not as good as that observed in the case of a 532-nm laser (see Fig. 2a). The performance of the sensor systems with different lasers is actually determined by many factors such as the spectral characteristics of the laser systems, the optics, and also the scattered light. Due to the broadband spectral line width of the 670-nm laser diode, a cut-off filter, centered on $725-\mathrm{nm}$, is unable to suppress the scattered 
incident laser light. The scattered light has contributed to part of the strong background we observed. The optical fiber can also give rise to its Raman spectral feature and fluorescence background signal that will superimpose on the $\mathrm{LOX}$ and $\mathrm{LN}_{2}$ spectrum. Our current prototype sensor is very compact, and we are unable to effectively remove these background features. To suppress the background and to improve the signal/noise ratio of the Raman signal, appropriate combinations of various filters will need to be tested and added to the collection optics of the miniaturized prototype sensor. However, we can apply some data processing technique to improve the quality of the data. Figure 5(b) shows the processed Raman LOX and $\mathrm{LN}_{2}$ spectrum of the prototype sensor. This spectrum was obtained by subtracting the raw data from the background spectrum (the background spectrum was obtained by fitting with some polynomial functions). The spectral averaging can also be applied to further improve the signal-to-noise ratio. Intensity ratios for a $\mathrm{LN}_{2}$ and $\mathrm{LOX}$ weight ratios of 1.5 were calculated to be $\sim 1.4$ from background subtracted spectrum obtained from the prototype sensor. The Intensity ratios obtained with a 532-nm laser for the same weight ratios of $\mathrm{LN}_{2} / \mathrm{LOX}$ is about 1.3.

\section{Conclusions}

This paper presents a consistent optical fiber Raman sensor for cryogenic applications and the evaluation of sensor performance with different excitation light sources. The sensor performance with two different 532-nm lasers and an Inphotonic probe was employed. Efficient light collection optics and good signal-to-noise ratio of Raman $\mathrm{LN}_{2} / \mathrm{LOX}$ signal make these laser-based sensor systems an effective tool for rapid as well as sensitive monitoring of $\mathrm{LN}_{2} / \mathrm{LOX}$ during the testing of a rocket engine. To design a low-cost, reliable sensor for this type of application, we have also tested with a compact and an inexpensive diode laser. Although this miniaturized prototype sensor system yielded a low signal-to-noise ratio of Raman 
$\mathrm{LN}_{2} / \mathrm{LOX}$ signal, we have shown that with an appropriate data processing technique, the extracted intensity ratio of Raman signal of $\mathrm{LN}_{2}$ and $\mathrm{LOX}$ are close to that obtained from the more-expensive 532-nm excitation systems. The miniaturized sensor system offers a great deal of flexibility and provides a cost effective solution for measuring the quality as well as quantity of cryogenic fluids. However, due to the relatively poor signal-to-noise ratio and background problem, the performance of the miniaturized sensor system is not suitable for real time monitoring. On the other hand, the system with a high-power 532-nm laser can offer good quality $\mathrm{LOX} / \mathrm{LN}_{2}$ spectra with reasonable response time, and therefore it is more suitable for real-time application. Future research will be focussed on improving the performance of the sensor for high cryogenic liquids pressure.

\section{Acknowledgments}

The authors gratefully acknowledge Dr. William St. Cyr and Mr. Tracy Miller for their support and Mr. R. Arun Kumar for advising on mixture measurement. This work is funded by NASA/SSC -STTR Contract No. NNS04AA34C through Mississippi Ethanol LLC.

\section{References}

1. R.O Ballard, "Fiber optic sensors for rocket engine applications"; presented at the $28^{\text {th }}$ Joint Propulsion Conference and Exhibit, Nashville, TN,6-8 July 1992.

2. Shin-Juh Chen and Joel A. Silver, "Detection of explosive mixtures in the ullage of aircraft fuel tanks", presented at the $42^{\text {nd }}$ Aerospace Meeting and Exhibit, Reno, Nevada, 5-8 Jan. 2004. 
3. R. Parry, G. Claborn, A. Haas, R. Landis, W. Page, J. Smith ,"A high reliability oxygen deficiency monitoring system"; presented at the Particle Accelerator Conference, Washington D.C., 17-20 May 1993.

4. M.A Nurge, R.Youngquist, D.Walters, "Capacitance based mass metering for cryogenic fluids", Cryogenics, 43, 501-506 (2003).

5. J.A. Akintunde, "Simple liquid nitrogen level monitor using copper wire as a sensor", Cryogenics, 27,266-268 (1987).

6. A.M.Domashenko and Y.A.Kondrashkov, "Technology of quality control of liquefied methane-A fuel for space rocket systems", Chemical \& Petroleum Eng., 39,656-661 (2003)

7. K.T.V Grattan and B.T. Meggitt, Optical fiber Sensor Technology, (Kluwer Academic Publishers, Boston, 1999)

8. J.P. Singh and F.Y.Yueh, "Real time, non-intrusive detection of liquid nitrogen in liquid oxygen at high pressure and high flow", NASA/SSC Final Report, Contract No. NNS04AA34C (2004).

9. Vidhu S. Tiwari, Sunil K. Khijwania, Fang-Yu Yueh, and Jagdish P. Singh, "Monitoring $\mathrm{N}_{2}$ \& $\mathrm{O}_{2}$ concentration ratios in a high-pressure gas mixture by fiber optic Raman Sensor", in Proceedings of Seventh International Conference on Optoelectronics, Fiber-optics and Photonics. (Cochin, India, 2004), pp.132-137.

10. D. Lin-Vien, N. B. Colthup, W. G. Fateley, and J. G. Grasselli, The Handbook of Infrared and Raman Characteristic Frequencies of Organic Molecules, (Academic Press, Boston, 1991.)

11. B. Schrader, Raman and Infrared Atlas of Organic Compounds, 2nd ed. (Wiley-VCH, Weinheim, 1989). 
12. R. A. Nyquist, C. L. Putzig, M. A. Leugers, and R.O. Kagel, The Handbook of Infrared and Raman Spectra of Inorganic Compounds and Organic Salts, vol. 1-4 (Academic Press, San Diego, 1997).

13. R. A. Dluhy, S. M. Stephens, S. Widayati, and A.D. Williams, "Vibrational spectroscopy of biophysical monolayers. Applications of IR and Raman spectroscopy to biomembrane model systems at interfaces" Spectrochim. Acta. Part A: Molecular and Biomolecular Spectrosc., 51, 1413-1447 (1995).

14. A.C. Eckbreth, Laser Diagnostics for Combustion Temperature and Species, 2nd ed. (Gordon and Breach, Amsterdam, 1996), Chap.5 and references therein, pp.209-280.

15. K. K. Kuo and T. P. Parr, eds., Non-intrusive Combustion Diagnostics, (Begell House, New York, 1994).

16. D. A. Long, Raman Spectroscopy ( McGraw-Hill, New York, 1977).

17. N. M. Laurendeau, "Temperature measurements by light-scattering methods", Prog. Energy Combust. Sci., 14, 147-170 ( 1998).

18. G. Zikratov, F.Y. Yueh, J. P. Singh, O. P. Norton, R.A. Kumar, and R.L. Cook, "Spontaneous anti-stokes Raman probe for gas temperature measurements in industrial furnaces", Appl. Opt.38, 1467-1475 (1999).

19. K. Kamogawa and T. Kitagawa, "Evidence for direct intermolecular interactions as an origin of the hydration shifts of the C-H stretching vibrations: 1, 4-dioxane/water system", Chem. Phys. Lett., 179, 271-276 (1991).

20. Tuan Vo-Dinh, Biomedical Photonics Handbook (CRC Press, Boca Raton, 2003).

21. Michael J. Pelletier, Analytical Applications of Raman Spectroscopy (Blackwell Science, Malden, 1999). 
22. Peter Atkins and Julio de Paula, Physical Chemistry (W.H.Freeman and Company, NewYork, 2003).

23. Herzberg, Spectra of Diatomic Molecules, 2nd ed (Van Nostrand Reinhold, New York, 1950). 


\begin{tabular}{cccccc}
\hline $\begin{array}{c}\text { Excitation } \\
\text { Source }\end{array}$ & $\begin{array}{c}\text { Wavelength } \\
(\mathrm{nm})\end{array}$ & $\begin{array}{c}\text { Laser Line } \\
\text { Width } \\
(\mathrm{nm})\end{array}$ & $\begin{array}{l}\text { Max. } \\
\text { Power } \\
(\mathrm{mW})\end{array}$ & $\begin{array}{c}\text { Beam } \\
\text { Diameter* } \\
(\mathrm{mm})\end{array}$ & $\begin{array}{c}\text { Transverse } \\
\text { Mode }\end{array}$ \\
\hline $\begin{array}{c}\text { A. 532-nm laser } \\
\text { Coherent }\end{array}$ & 532 & 0.00047 & 330 & 0.70 & TEM $_{00}$ \\
Milles Griot & 532 & 0.18 & 3000 & 0.24 & Multimode \\
$\begin{array}{l}\text { B.670-nm diode laser } \\
\text { Power } \\
\text { Technology Inc. }\end{array}$ & 670 & 0.70 & 700 & 1.5 & Multimode \\
\hline
\end{tabular}

Table 1. The excitation sources used in this study

$* 1 / \mathrm{e}^{2}$ at waist

\begin{tabular}{ccc}
\hline & 532-nm Laser & 670-nm Diode Laser \\
\hline Maximum Laser Power on Sample & $140 \mathrm{~mW}$ & $380 \mathrm{~mW}$ \\
CCD Detector Efficiency & 2.3 & 0.4 \\
Grating Efficiency & $60 \%$ & $72 \%$ \\
Relative Raman Cross Section $\left(\lambda^{-4}\right)$ & 12.48 & 4.96 \\
Overall Relative Raman signal & 4.44 & 1 \\
\hline
\end{tabular}

Table 2. Estimation of the Raman Signal from 532-nm and 670-nm laser 


\section{List of Figure Captions}

Fig. 1. Experimental set-up of optical fiber Raman sensor. (a) InPhotonic Raman Probe based system (b) Reflection probe based prototype Raman system.

Fig. 2. (a) Raman Spectrum of $40 \% \mathrm{LOX}$ and $60 \% \mathrm{LN}_{2}$ liquid mixture. Laser wavelength: $532 \mathrm{~nm}$

Fig. 2 (b) Sensor response to the liquid $\mathrm{N}_{2} / \mathrm{O}_{2}$ mixture as a function of $\mathrm{LN}_{2}$ concentration.

Fig. 3 Sensor response to the liquid $\mathrm{N}_{2} / \mathrm{O}_{2}$ mixture as a function of $\mathrm{LN}_{2} / \mathrm{LOX}$ weight ratio.

Fig. 4. The area ratio variation of $\mathrm{LN}_{2} / \mathrm{O}_{2}$ against their weight ratios.

Fig. 5 Raman spectrum for $60 \% \mathrm{LN}_{2} \& 40 \% \mathrm{LOX}$ with $670 \mathrm{~nm}$ diode laser (a) actual spectrum (b) background subtracted spectrum 
(a)

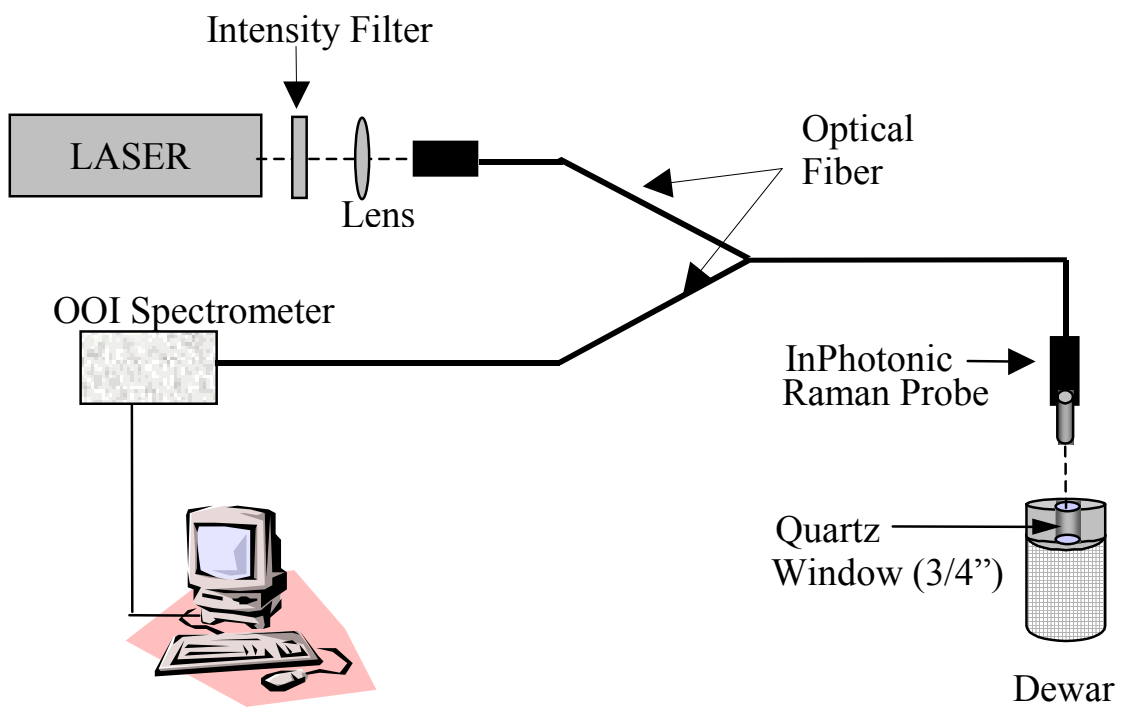

(b)

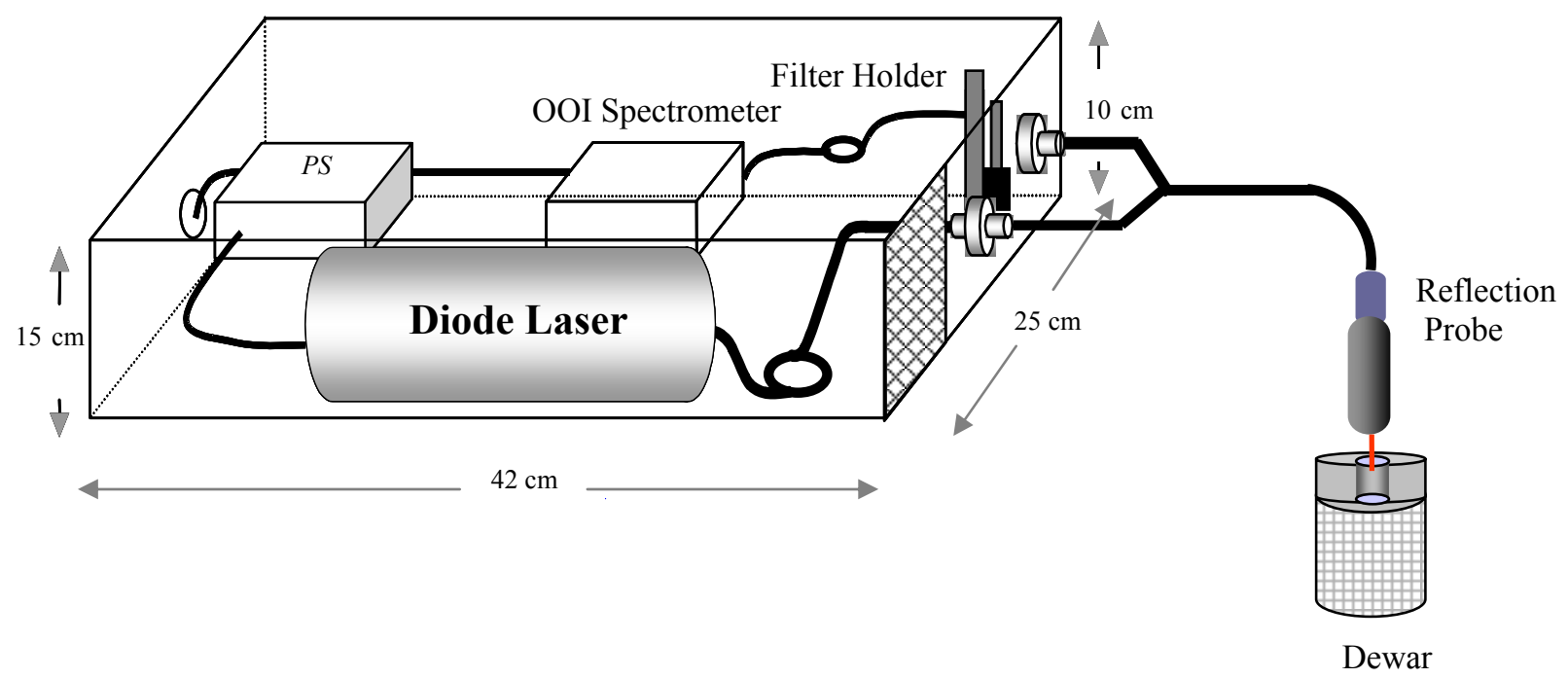

Fig. 1. Experimental set-up of optical fiber Raman sensor. (a) InPhotonic Raman Probe based system (b) Reflection probe based prototype Raman system. 


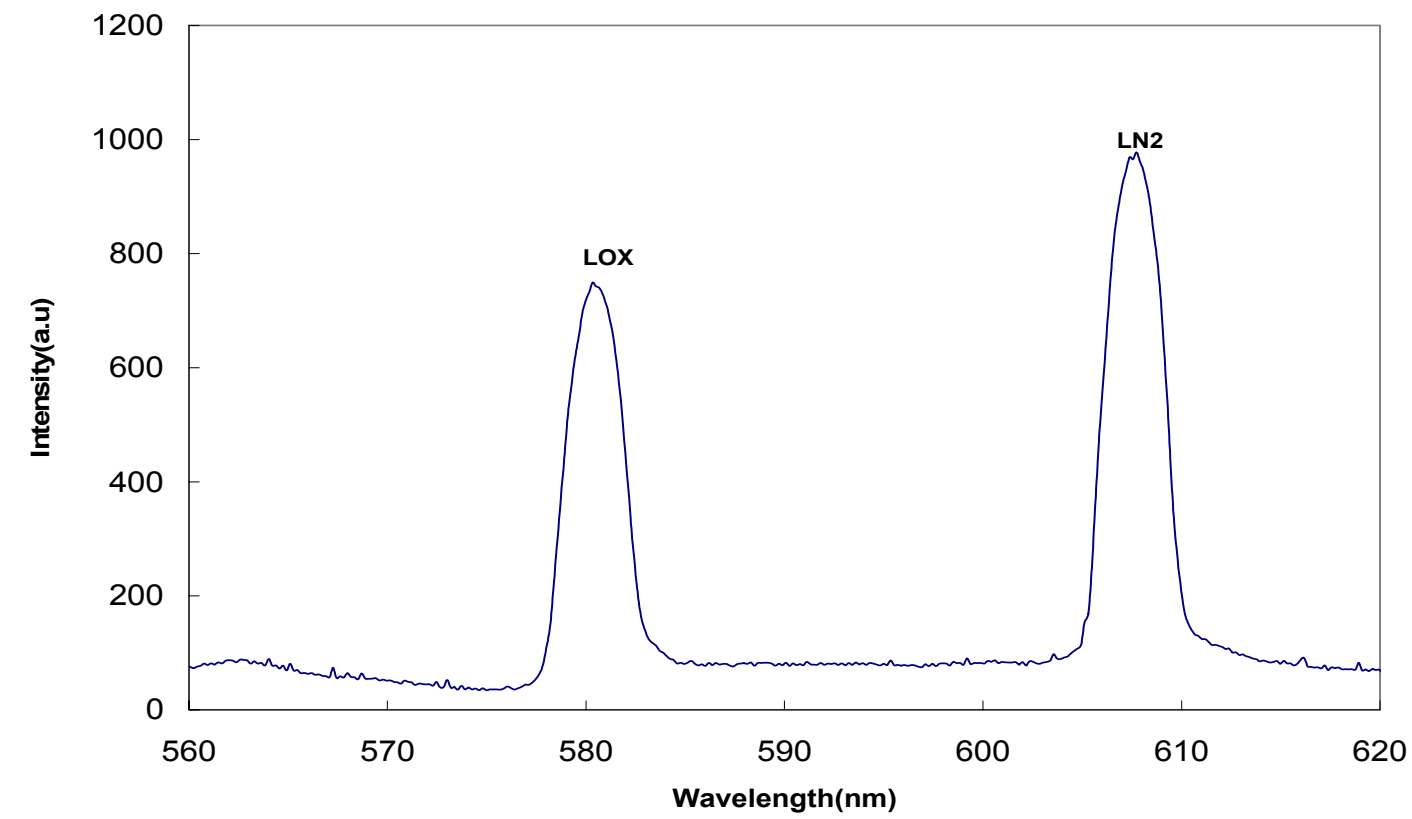

Fig. 2. (a) Raman Spectrum of $40 \% \mathrm{LOX}$ and $60 \% \mathrm{LN}_{2}$ liquid mixture. Laser wavelength: $532 \mathrm{~nm}$

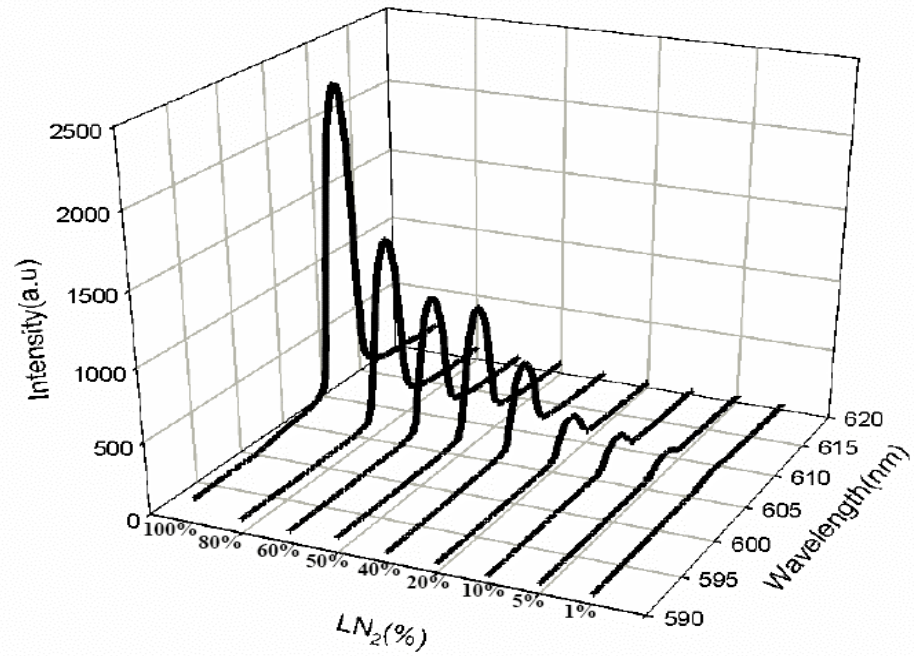

Fig. 2 (b) Sensor response to the liquid $\mathrm{N}_{2} / \mathrm{O}_{2}$ mixture as a function of $\mathrm{LN}_{2}$ concentration. 


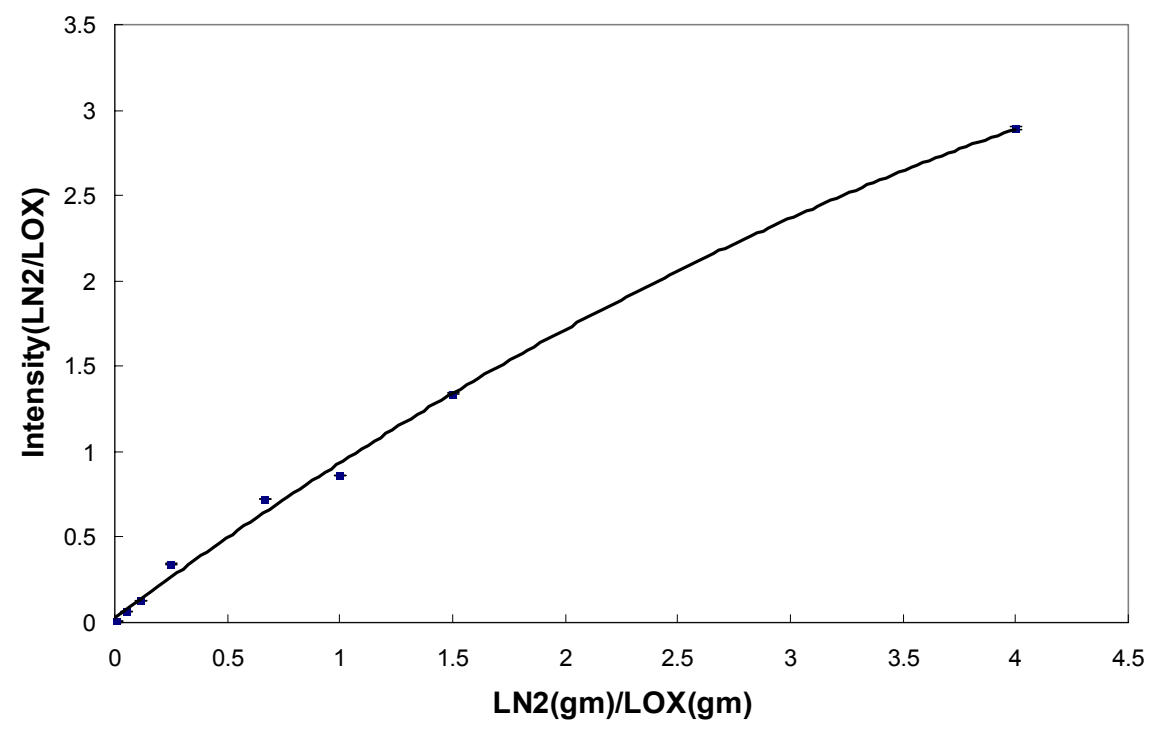

Fig.3 Sensor response to the liquid $\mathrm{N}_{2} / \mathrm{O}_{2}$ mixture as a function of $\mathrm{LN}_{2} / \mathrm{LOX}$ weight ratio. 


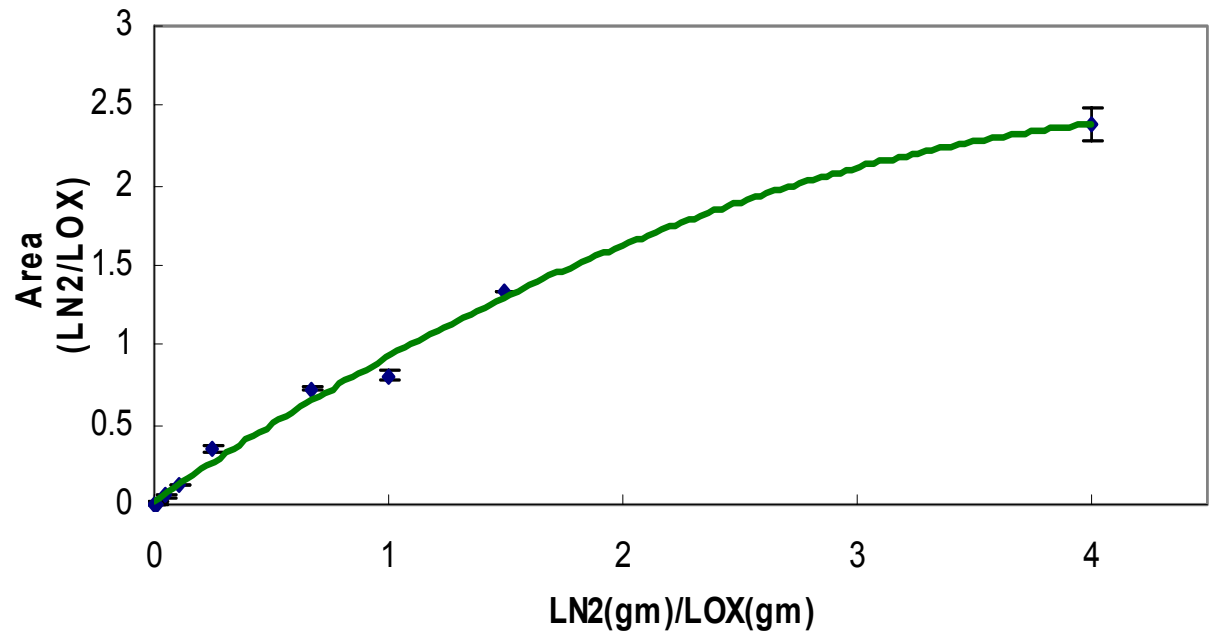

Fig. 4. The area ratio variation of $\mathrm{LN}_{2} / \mathrm{O}_{2}$ against their weight ratios. 

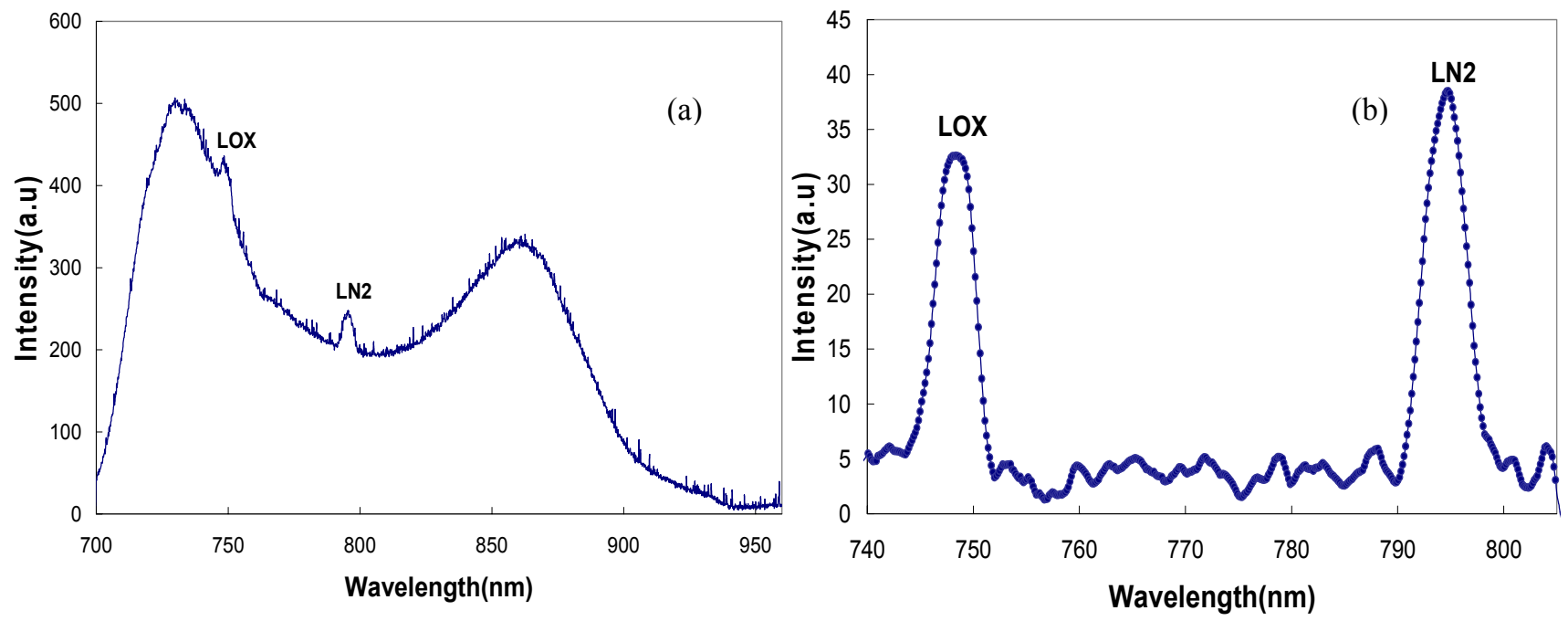

Fig. 5 Raman spectrum for $60 \% \mathrm{LN}_{2} \& 40 \% \mathrm{LOX}$ with $670 \mathrm{~nm}$ diode laser (a) actual spectrum (b) background subtracted spectrum 